\title{
28 Research Square \\ PARP1 Bound to XRCC2 Promotes Tumor Progression in Colorectal Cancer
}

\author{
Kaiwu Xu \\ Hunan Provincial People's Hospital \\ Zhige Yu \\ Hunan Provincial People's Hospital \\ Tailiang Lu \\ Hunan Provincial People's Hospital \\ Wei Peng \\ Hunan Provincial People's Hospital \\ Yongqiang Gong \\ Hunan Provincial People's Hospital \\ Chaowu Chen ( $\square$ chenchaowu0@163.com ) \\ Hunan Provincial People's Hospital https://orcid.org/0000-0001-5912-9050
}

\section{Research}

Keywords: PARP1, XRCC2, Progression, Colorectal cancer, Gastrointestinal

Posted Date: February 4th, 2021

DOI: https://doi.org/10.21203/rs.3.rs-176757/v1

License: (c) (i) This work is licensed under a Creative Commons Attribution 4.0 International License.

Read Full License 


\section{Abstract}

Background: By complexing poly (ADP-ribose) (PAR) in reaction to breaked strand, PAR polymerase1 (PARP1) acts as the key enzyme participated in DNA repair. However, recent studies suggest that unrepaired DNA breaks results in persistent PARP1 activation, which leads to a progressively reduce in hexokinase1 (HK1) activity and cell death. So the molecular mechanism of PARP1 remains elusive.

Methods: 212 colorectal cancer (CRC) patients who had the operation at our hospital were recruited. Used immunohistochemistry to evaluate PARP1 expression. Survival analysis was calculated based on PARP1 expression.

Results: Compared with matching adjacent noncancerous tissue, in CRC tissue, PARP1 expression was remarkably higher, which was correlated with the degree of differentiation, TNM stage, depth of invasion, distant metastasis, and 5-year survival. Furthermore, after constructing CRC cell lines stably expressing low or high PARP1, we found that PARP1 overexpression promoted proliferation, and proved PARP1 interacted with XRCC2 in CRC cells by immunoprecipitation (IP) analysis.

Conclusions: PARP1 was upregulated in CRC cells and promoted its proliferation of colorectal cancer cells. Furthermore, PARP1 expression status was significantly related to some clinicopathological features and 5-year survival.

\section{Introduction}

Colorectal cancer (CRC), which ranks fourth (6.1\%) in the light of incidence but second $(9.2 \%)$ in accordance with mortality in diagnosed cancer, takes a place of the most usual gastrointestinal cancers worldwide. Currently, in 2018, around 1.8 million people were confirmed CRC worldwide, and over 881,000 patients died of the disease, occupying about $10 \%$ cancer cases and deaths. ${ }^{1}$ More importantly, in recent decades, the CRC incidence and mortality have increased in China. ${ }^{2}$ Scholars have made progress in targeted therapy of CRC; nevertheless, superior targeting drugs are desired since the current treatment cannot generate satisfactory results.

Changes in PARP1 levels play a vital role in CRC. ${ }^{3}$ PARP1 plays a fundamental role in preserving genome stability and regulating chromatin structure. PARP1 takes part in several DNA repair pathways, which include DNA single-strand break (SSB) repair, HR repair (HRR) pathway of DNA double-stranded breaks (DSBs), and base excision repair (BER). HRR-deficient neoplasms are extremely susceptive to PARP1 inhibitors based on the synthetic lethality theory. ${ }^{4-6}$ PARP1 inhibitors chiefly prevent the PARP1 catalytic activity and raise the single-strand breaks levels persistently, resulting in DNA DSBs upon replication. ${ }^{5,7,8}$ On the other hand, unrepaired DNA breaks, arising from DNA repair deficiency and/or overexposure of genotoxin, results in persistently PARP1 activated and cell death. ${ }^{9}$ Uncontrolled or excessive activation of PARP1 leads to numerous pathological results, including the onset of diabetes, streptozotocin-induced pancreatic beta-cell death, myocardial ischemia, and tissue injury from cerebral. ${ }^{10-13}$ What's more, the 
study of Fouquerel ${ }^{14}$ has demonstrated that by inhibiting HK1 independent of NAD + depletion, PARP1 regulates glycolysis negatively. The result showed that activated PARP1 inhibited the activity of HK1, which resulted in the inhibition of ATP synthesis and cell death.

Therefore, PARP1 as an important member of DNA damage repair and energy metabolism pathway of tumor cells, its specific role and mechanism have not been clarified. In this research, we intend to study the effect of PARP1 in CRC cells and provide an important basis for PARP1 to become a new therapeutic target for CRC.

\section{Methods}

\section{Patients}

From October 2010 to December 2012, 212 primary CRC tissues and 47 matching adjacent noncancerous tissues were obtained from patients who had the operation at the Hunan Provincial People's Hospital. Patients with preoperative chemotherapy and/or radiotherapy, and palliative surgery were excluded. Survival analysis excluded patients who died within 30 days after surgery, since their death could be attributed to surgical complications. All patients have signed an informed consent. The Ethical Review Board of Hunan Provincial People's Hospital have approved the study.

\section{Immunohistochemistry (IHC)}

According to previously described methods, we performed the following procedures by the classic biotinstreptavidin-peroxidase $\mathrm{IHC}$ staining protocols. ${ }^{15}$ We obtained sections from the Hunan Provincial People's Hospital Pathology Department, and incubated overnight at $48^{\circ} \mathrm{C}$ with polyclonal primary antibody against PARP1 (1:100; Abcam, Cambridge, UK). Following incubated with diaminobenzidine and horseradish peroxidase-conjugated sheep anti-rabbit secondary antibody (Beyotime; Guangzhou, China), used Mayer's hematoxylin to counterstain the slides. Positive control was primary CRC tissue slides. In negative control staining, used phosphate-buffered saline (PBS) buffer to replace the primary antibody. The immunostaining results were scored according to the methods previously described below. ${ }^{16}$

\section{Culture and treatment of Cell}

We obtained human CRC cell lines FHC, SW480, SW620, LoVo, SW403, HT-29, COLO205 and COLO320DM from the American Type Culture Collection (Manassas, VA, USA). Incubated cell lines in DMEM/RPMI1640 medium (Gibco, Thermo Fisher Scientific, Waltham, MA, USA) complemented with penicillin $(100 \mathrm{U} / \mathrm{mL})$, streptomycin $(100 \mu \mathrm{g} / \mathrm{ml})$, and 10\% FBS (Gibco, Thermo Fisher Scientific, Waltham, MA, USA) at $37^{\circ} \mathrm{C}$ with $5 \% \mathrm{CO}_{2}$.

\section{Vectors and retroviral infection}

Through subcloning the PCR-amplified human PARP1 coding sequence into a pBABE-puro vector, we generated pBABE/PARP1-overexpressing human PARP1. Cloned two RNA interference (RNAi) 
oligonucleotides into pSuper-retro-puro vectors to produce the pSuper-retro-PARP1-RNAi respectively, thereby silencing endogenous PARP1. As previously described, the generation and infection of retroviruses were carried out. ${ }^{17}$ After infection 48 hours, cell line stably expressing PARP1 (pBABE-puroPARP1 or SW480/SW620-PARP1; control, pBABE-puro or SW480/SW620-Vector, respectively) or PARP1 RNAi (pSuper- retro-puro-si PARP1 or SW480/SW620-PARP1/RNAi; control, pSuperretro-puro or SW480/SW620-Scramble, respectively) were selected using puromycin $(0.5 \mathrm{mg} / \mathrm{ml})$ over 10 days. SDSPAGE was used to segregate SW480 and SW620 cell lysates to detect PARP1 protein levels.

\section{Extraction and reverse transcription of RNA, real-time quantitative PCR}

In line with manufacturer's illustrations, applied Trizol reagent (Invitrogen, Carlsbad, CA, USA) to accomplish total RNA extraction from cultured cells or tissues. Equipped with the ABI PRISM 7500 system (Applied Biosystems, Foster City, CA, USA), SYBR Green I (Invitrogen) was applied for real-time quantitative PCR. Selected housekeeping gene GAPDH as an internal control. Used the primers below:

PARP1 forward, 5'-ACAGTGTGCAGGCCAAGGTG -3', and reverse 5'-CTCGGC TTCTTCAGAATCTCTGTC-3'; XRCC2 forward: 5'-TCACCTGTGCATGGTG ATATT-3', and reverse: 5'-TTCCAGGCCACCTTCTGATT-3'; GAPDH forward: 5'-GACTCATGACCACAGTCCATGC-3', and reverse: 5'-AGAGGCAGGGATGATG

TTCTG-3'; p21 forward: 5'-CGATGCCAACCTCCTCAACGA-3', and reverse: 5'-TCGCAGACCTCCAGCATCCA3'; cyclin D1 forward: 5'-AACTACCTGGA CCGCTTCCT-3', and reverse: 5'-CCACTTGAGCTTGTTCACCA-3'.

\section{Western blotting}

According to manufacturer's instructions $\llbracket$ proteins were prepared from cell lysates, isolated on SDS-PAGE, and transferred to PVDF membranes. To detect specific proteins, primary antibodies that were used included a-Tubulin mouse monoclonal antibody (1:1000; Sigma-Aldrich, St. Louis, MO, USA), anti-human XRCC2 mouse monoclonal antibody (1:1500; Abcam), anti-human PARP1 mouse monoclonal antibody (1:1500; Abcam), anti-human cyclin D1 rabbit monoclonal antibody (1:1500; Abcam), and anti-human P21 rabbit monoclonal antibody (1:1500; Abcam). The secondary antibody was goat anti-mouse antibody (1:2000; Santa Cruz Biotechnology, Santa Cruz, CA, USA). After the membrane exposed to electrochemiluminescence reagent (GE Healthcare, Buckinghamshire, UK), storm imaging system (Amersham Biosciences, Piscataway, NJ, USA) was used for visualization to achieve signal amplification and detection.

\section{Cell proliferation detection}

In accordance with the manufacturer's illustrations, used the Cell Counting Kit-8 (CCK-8) cell proliferation kit (Dojindo Laboratories, Kumamoto, Japan) to assess cell proliferation. Concisely, seeded the cells into 96-well plates $\left(2 \times 10^{3}\right.$ cells/well), and cultured under regular circumstances with $100 \mu \mathrm{L}$ complete medium. At the specified time, incubated cells with RPMI-1640 medium $(100 \mu \mathrm{L})$ plus CCK8 reagent 
$(10 \mu \mathrm{L})$ for 2 hours at $37^{\circ} \mathrm{C}$. After that, measured the absorbance at $450 \mathrm{~nm}$ wavelength on a microplate reader (Bio-Rad, La Jolla, CA, USA). Conducted three repetition experiments independently.

\section{Colony formation assay}

In brief, plated exponential growth cells into 6-well plates at 1000 cells/well and cultured for 10-14 days at $37^{\circ} \mathrm{C}$ with $5 \% \mathrm{CO} 2$. For visualization and counting, used $75 \%$ ethanol to fix the colonies for $30 \mathrm{mins}$ and stained with $0.5 \%$ crystal violet (Beyotime, Nanjing, China) afterwards. When colonies with more than 50 cells would be manually calculated. Every group of cells comprised three wells, and three independent repeat experiments were conducted.

\section{Statistical analysis}

SPSS 20.0 (SPSS Inc, Chicago, IL, USA) was used for statistic analysis. Employed the Chi-square test to evaluate the association between PARP1 expression and clinicopathological characteristics. The significant differences between two groups of data were analyzed with the Student's t test. The log-rank test and the Kaplan-Meier method were employed for survival curves analysis. The time from surgery to last follow-up date or patient's death was 5-year overall survival (OS). The time from radical operation to recurrence, last follow-up date, or death was defined as Relapse-free survival (RFS). Recurrences were defined as local and distant relapses. Statistically significant was set at $p<0.05$.

\section{Results}

\section{In CRC cell lines and clinical tissues PARP1 was upregulated.}

PARP1 expression in primary CRC tissue and matching adjacent noncancerous tissue was described in Fig. 1. In 157 of 212 (74.1\%) primary CRC tissues, positive PARP1 staining was discovered. By contrast, compared with primary CRC tissue,in the matching adjacent noncancerous tissues, PARP1 staining positive rate was only 53.2\% (25/47 samples, $p=0.005$; Fig. 1A-D; Table 1$)$. Furthermore, applied western blotting and real-time PCR to detect eight CRC patients PARP1 expression in tumors and the matching adjacent noncancerous tissues. Different from normal tissues, PARP1 was upregulated remarkably in tumors ( $p<0.05$; Fig. 2A, B). Moreover, we also used western blotting and real-time PCR to examine PARP1 expression in normal colonic epithelial cell (FHC) and eight CRC cell lines. Compared with colonic epithelial cell, PARP1 was upregulated remarkably in CRC cell lines $(p<0.05$; Fig. 2 C, D).

Table 1

PARP1 expression in primary CRC tissue and adjacent noncancerous tissue.

\begin{tabular}{|lllll|}
\hline Tissue sample & $\mathrm{n}$ & \multicolumn{2}{l}{ Expression of PARP1 } & \multirow{2}{*}{$p$} \\
\cline { 3 - 4 } & & Positive (\%) & Negative (\%) & \\
\cline { 1 - 4 } Primary colorectal cancer & 212 & 157(74.1) & $55(25.9)$ & \multirow{2}{*}{0.005} \\
\cline { 1 - 4 } Adjacent normal colorectal mucosa tissues & 47 & $25(53.2)$ & $22(46.8)$ & \\
\hline
\end{tabular}




\section{Correlation between PARP1 expression and CRC patients clinicopathological characteristics.}

Table 2 described the relevance between PARP1 expression and clinicopathological features, including age, gender, differentiation, distant metastasis, tumor-nodes-metastasis (TNM) stage, tumor size, lymph node metastasis, tumor site, and depth of invasion. The expression status of PARP1 in primary CRC was remarkably associated with the degree of differentiation, TNM stage, distant metastasis, and depth of invasion ( $p=0.015 ; p=0.002 ; p=0.001 ; p=0.001$, respectively). 
Table 2

Clinicopathological characteristics and PARP1 expression status of patients with colorectal cancer.

\begin{tabular}{|c|c|c|c|c|}
\hline Clinical characteristics & $n$ & Positive (\%) & Negative (\%) & $p$ \\
\hline \multicolumn{5}{|l|}{ Gender } \\
\hline male & 102 & 73(71.6) & $29(28.4)$ & 0.426 \\
\hline female & 110 & $84(76.4)$ & $26(23.6)$ & \\
\hline \multicolumn{5}{|l|}{ Age } \\
\hline$<50 y$ & 55 & $44(80.0)$ & $11(20.0)$ & 0.503 \\
\hline $50-70 y$ & 122 & $88(72.1)$ & $34(27.9)$ & \\
\hline$>70 y$ & 35 & $25(71.4)$ & 10(28.6) & \\
\hline \multicolumn{5}{|l|}{ Tumor site } \\
\hline rectum & 83 & $60(72.3)$ & $23(27.7)$ & 0.830 \\
\hline left & 85 & $63(74.1)$ & $22(25.9)$ & \\
\hline right & 44 & $34(77.3)$ & $10(22.7)$ & \\
\hline \multicolumn{5}{|l|}{ Tumor size } \\
\hline$<3 \mathrm{~cm}$ & 24 & 17(70.8) & $7(29.2)$ & 0.149 \\
\hline $3-5 \mathrm{~cm}$ & 111 & $77(69.4)$ & $34(30.6)$ & \\
\hline$>5 \mathrm{~cm}$ & 77 & 63(81.8) & $14(18.2)$ & \\
\hline \multicolumn{5}{|l|}{ Lymph node metastasis } \\
\hline Yes & 144 & 112(77.8) & $32(22.2)$ & 0.072 \\
\hline No & 68 & $45(66.2)$ & 23(33.8) & \\
\hline \multicolumn{5}{|l|}{ Distant metastasis } \\
\hline Yes & 54 & $49(90.7)$ & $5(9.3)$ & * 0.001 \\
\hline no & 158 & 108(68.4) & $50(31.6)$ & \\
\hline \multicolumn{5}{|l|}{ Depth of invasion } \\
\hline T1 & 8 & $3(37.5)$ & $5(62.5)$ & * 0.001 \\
\hline $\mathrm{T} 2$ & 20 & $9(45.0)$ & $11(55.0)$ & \\
\hline T3 & 45 & $33(73.3)$ & $12(26.7)$ & \\
\hline
\end{tabular}




\begin{tabular}{|lllll|}
\hline Clinical characteristics & $\mathbf{n}$ & Positive (\%) & Negative (\%) & $\boldsymbol{p}$ \\
\hline T4 & 139 & $112(80.6)$ & $27(19.4)$ & \\
\hline Degree of differentiation & & & & \\
high & 4 & $1(25.0)$ & $3(75.0)$ & $* 0.015$ \\
\hline moderately & 161 & $116(72.0)$ & $45(28.0)$ & \\
\hline low & 47 & $40(85.1)$ & $7(14.9)$ & \\
\hline TNM & & & & \\
\hline I/II & 37 & $20(54.1)$ & $17(45.9)$ & $* 0.002$ \\
\hline III/IV & 175 & $137(78.3)$ & $38(21.7)$ & \\
\hline$* p<0.05$. & & & & \\
\hline
\end{tabular}

\section{Survival analysis and prognostic significance of PARP1 expression.}

Figure 3A, B showed the Kaplan-Meier estimates for the group with positive PARP1 and group with negative PARP1. The median time to total OS for 212 patients was 43.9 months. And the median OS time for two groups was 40.7 months and 53.1 months separately. The two survival curves were remarkably different (Fig. 3A; $\left.\chi^{2}=12.095 ; p=0.001\right)$. 212 patients total median RFS time was 38.0 months. While the median RFS time for two groups was 34.7 months and 48.1 months respectively. The two survival curves were also significantly different (Fig. $3 \mathrm{~B} ; \chi^{2}=10.848 ; p=0.001$ ). These results showed that, compared with negative PARP1 expression, patients with positive PARP1 expression had shorter OS and RFS.

\section{Stable cell lines expressing high or low PARP1.}

Western blotting and quantitative PCR (QPCR) was used to detect PARP1 expression after cell lines expressing high or low PARP1 were constructed. Western blotting revealed that PARP1 expression in SW480/SW620-PARP1/RNAi was lower than that in SW480/SW620-Scramble cells. What's more, PARP1 expression in SW480/SW620-PARP1 cells was higher than that in SW480/SW620-Vector cells (Fig. 4B; $p$ $<0.05$ ). Moreover, the results obtained by QPCR were similar (Fig. 4A; $p<0.05$ ). The above results demonstrated that cell lines stably expressed low or high PARP1 had been constructed successfully.

\section{PARP1 interacted with XRCC2 during SW620 cell proliferation.}

In previous studies, we found that the polymorphism or low expression of XRCC2 affected the sensitivity of CRC cells to PARP1 inhibitors ${ }^{15,18}$. In the light of previous theory, we assumed that PARP1 binds to XRCC2 in SW620 cells. To define the combination between PARP1 and XRCC2, IP was performed. Using PARP1 and XRCC2 primary antibodies to perform two-way verification, both of which showed that, during SW620 cell differentiation, PARP1 interacted with XRCC2 (Fig. 4C). 
PARP1 overexpression promoted proliferation in CRC cells.

Through CCK-8 assay and colony formation assay, we assessed PARP1 overexpression effect on CRC cell proliferation to study whether PARP1 affects CRC development and progression. Compared with PARP1/RNAi, PARP1 upregulation remarkably increased SW480/SW620 cells growth rate 48 hours after PARP1 transduction (Fig. 5A, B; $p<0.05$ ). As expected, PARP1 overexpression increased the expression of cyclin D1, while significantly reducing p21 expression in CRC cells (Fig. $5 C, D ; p<0.05$ ). The results demonstrate that PARP1 may make a significant impact in CRC cells proliferation.

\section{Discussion}

These traditional views believe that PARP1 had biological functions. For instance, DNA repair, apoptosis, synthetic lethality, histone binding, necrosis, and so on. ${ }^{19}$ After activated by DNA damage, PARP1 startup the DNA damage repair process as a tumor suppressor gene, binds to target proteins, DNA signal- and double- strand breaks, and other proteins of DNA repair. ${ }^{20}$ However, the latest research showed that PARP1 participated nucleus to mitochondria communication, which involved in cell energy metabolism. Activation of PARP1 sparks off cell death and energy crumble, which indicate novel understanding on the significance of PARP1 activation. Therefore, the role of PARP1 in cells is undefined. Current researches have pointed out that PARP1 may be related to the CRC occurrence. Studies have proved that PARP1 (Ala762Val, rs1136410) was associated with the susceptibility to colorectal carcinoma of adult and children. ${ }^{21,22}$ Moreover, the study of Sakthianandeswaren has found that MACROD2 deletions or haploinsufficient caused chromosome instability (CIN) and impaired PARP1 activity in CRC. In turn, it drove the evolution of cancer. ${ }^{23,24}$ Therefore, we focus on the significance of PARP1 in colorectal cancer.

By Immunohistochemistry, real-time PCR, and western blotting, compared with normal tissues, we discovered that PARP1 was upregulated significantly in tumors. These lead us to further study the significance of PARP1 in the development of CRC. Furthermore, after analyzing the correlation between PARP1 expression and the CRC clinicopathological features, we surprised to find that PARP1 expression status was remarkably correlated with the degree of differentiation, TNM stage, distant metastasis, and depth of invasion in primary CRC. Increasing PARP1 expression may promote invasive behavior and metastatic process of CRC. However, the mechanism of how PARP1 affects the progression of CRC remains unknown. Former researchers hold the assumption that PARP1 plays a vital part in facilitating the growth and proliferation of tumor cell. Santos $\mathrm{JC}^{25}$ revealed that the expression of PARP1 is related to tumor location (tumor of colon or rectum) and tumor stage (III/IV or I/II grade). Similarly, Li ${ }^{21}$ proved that PARP1 (Ala762Val) was associated with the susceptibility to CRC. Thus, it may not be surprising that PARP1 is involved in the metastasis and invasion of CRC. It worth further studies to clarify the mechanism involved.

What's more, in this research we found that the positive PARP1 expression and CRC patients' poor survival after surgery was correlated significantly. Patients with positive PARP1 expression had shorter OS and RFS compared with those patients with negative PARP1 expression. The two survival curves were 
remarkably different. The finding indicated that the PARP1 protein may affect the prognosis of CRC patients. However, the study of $\mathrm{Li}^{21}$ found that the mutation of PARP1 (Ala762Val) may have nothing to do with the prognosis of CRC patients intrinsically. Therefore, to define the relevance between PARP1 and CRC patients prognosis, expand the sample and enroll more advanced CRC patients is necessary.

To further figure out the effect of PARP1 in CRC progression and development, cell lines stably expressing high (SW480-PARP1, SW620-PARP1) or low PARP1 (SW480-PARP1/RNAi, SW620-PARP1/RNAi) were constructed. Firstly, we investigated the affection of high or low PARP1 expression on the growth of SW480/SW620 cells. Since one of the vital characteristics of the cancer cell phenotype is unconstrained growth. The current result revealed that suppressing PARP1 effectively decrease proliferation, whereas upregulating it significantly promote proliferation, indicating that PARP1 could be a vital regulator of CRC cell proliferation.

Schaaf ${ }^{26}$ found that PARP1 plays a vital part in the chemosensitization mechanism of hyperthermia of CRC. Previous study has pointed out that two genes simultaneously deficiencies lead biological systems lethality, otherwise the absence of one gene will be abided. ${ }^{25}$ Based on this concept, restraining PARP1 could be a latent therapeutic schedule for the therapy of cancers with defects in precise DNA repair genes, such as XRCC2, BRCA1/2, and MRE11. ${ }^{26,27}$ A number of clinical trials have been launched with PARP inhibitors apply to CRC patients. ${ }^{27}$ Furthermore, our study reveals that CRC cell proliferation restrained by olaparib in a dosage and time dependent way. ${ }^{15}$ These studies show that PARP1 expression can promote the proliferation of CRC cells. Moreover, PARP1 inhibitors have been shown to play an anti-tumor role in many tumors. ${ }^{15,28}$ Both PARP1 and XRCC2 participate in the double-stranded DNA HRR pathway. In the previous study, we found that CRC cell proliferation restrained by PARP1 inhibitor (olaparib) in a dosage and time dependent way. These findings indicated that there is a closed relationship between XRCC2 and PARP1. The effect of PARP1 inhibitor may require the presence of XRCC2. In the present study, we found that PARP1 interacted with XRCC2 in SW480/SW620 cells. However, up to now, there is no convinced proof to define the relevance between XRCC2 and PARP1. In future experiments, we will further explore the close relationship between PARP1 and XRCC2.

In conclusion, we found that PARP1 was upregulated in CRC and promotes colorectal cancer cells proliferation. Furthermore, PARP1 expression status in primary CRC was remarkably correlated with the degree of differentiation, TNM stage, distant metastasis, depth of invasion, and 5-year survival. Lastly, the results of IP show that XRCC2 protein interacting with PARP1, but need further explore.

\section{Declarations}

\section{Ethics approval and consent to participate}

All patients have signed an informed consent. The Ethical Review Board of Hunan Provincial People's Hospital have approved the study. 


\section{Consent for publication}

Not applicable.

\section{Availability of data and materials}

The datasets used and/or analysed during the current study are available from the corresponding author on reasonable request.

\section{Competing interests}

The authors declare that they have no competing interests.

\section{Funding}

This study was supported by grants from the Natural Science Foundation of Guangdong Province (2017A030310215), Medical Scientific Research Foundation of Guangdong Province (A2017267).

\section{Authors' contributions}

Kaiwu Xu: project development, data analysis and collection, manuscript writing and editing. Zhige $\mathrm{Yu}$, Tailiang Lu, Wei Peng, Yongqiang Gong: data collection. Chaowu Chen: Project design and quality control, manuscript editing.

\section{Acknowledgments}

Not applicable.

\section{References}

1. Bray F, Ferlay J, Soerjomataram I, Siegel RL, Torre LA, Jemal A. Global cancer statistics 2018 : GLOBOCAN estimates of incidence and mortality worldwide for 36 cancers in 185 countries. CA: a cancer journal for clinicians. 2018;68(6):394-424.

2. Arnold M, Sierra MS, Laversanne M, Soerjomataram I, Jemal A, Bray F. Global patterns and trends in colorectal cancer incidence and mortality. Gut. 2017;66(4):683-691.

3. Nosho K, Yamamoto H, Mikami M, Taniguchi H, Takahashi T, Adachi Y, et al. Overexpression of poly(ADP-ribose) polymerase-1 (PARP-1) in the early stage of colorectal carcinogenesis. European journal of cancer. 2006;42(14):2374-2381.

4. Bryant HE, Schultz N, Thomas HD, Parker KM, Flower D, Lopez E, et al. Specific killing of BRCA2deficient tumours with inhibitors of poly(ADP-ribose) polymerase. Nature. 2005;434(7035):913-917.

5. Farmer H, McCabe N, Lord CJ, Tutt AN, Johnson DA, Richardson TB, et al. Targeting the DNA repair defect in BRCA mutant cells as a therapeutic strategy. Nature. 2005;434(7035):917-921. 
6. Dedes KJ, Wilkerson PM, Wetterskog D, Weigelt B, Ashworth A, Reis-Filho JS. Synthetic lethality of PARP inhibition in cancers lacking BRCA1 and BRCA2 mutations. Cell cycle. 2011;10(8):1192-1199.

7. Hoeijmakers JH. DNA damage, aging, and cancer. The New England journal of medicine. 2009;361(15):1475-1485.

8. McAndrew EN, Lepage CC, McManus KJ. The synthetic lethal killing of RAD54B-deficient colorectal cancer cells by PARP1 inhibition is enhanced with SOD1 inhibition. Oncotarget. 2016;7(52):8741787430.

9. Tang JB, Goellner EM, Wang XH, Trivedi RN, St Croix CM, Jelezcova E, et al. Bioenergetic metabolites regulate base excision repair-dependent cell death in response to DNA damage. Molecular cancer research : MCR. 2010;8(1):67-79.

10. Burns N, Gold B. The effect of 3-methyladenine DNA glycosylase-mediated DNA repair on the induction of toxicity and diabetes by the beta-cell toxicant streptozotocin. Toxicological sciences : an official journal of the Society of Toxicology. 2007;95(2):391-400.

11. Masutani M, Suzuki H, Kamada N, Watanabe M, Ueda O, Nozaki T, et al. Poly(ADP-ribose) polymerase gene disruption conferred mice resistant to streptozotocin-induced diabetes. Proceedings of the National Academy of Sciences of the United States of America. 1999;96(5):2301-2304.

12. Eliasson MJ, Sampei K, Mandir AS, Hurn PD, Traystman RJ, Bao J, et al. Poly(ADP-ribose) polymerase gene disruption renders mice resistant to cerebral ischemia. Nature medicine. 1997;3(10):1089-1095.

13. Pieper AA, Walles T, Wei G, Clements EE, Verma A, Snyder SH, et al. Myocardial postischemic injury is reduced by polyADPripose polymerase-1 gene disruption. Molecular medicine. 2000;6(4):271-282.

14. Fouquerel E, Goellner EM, Yu Z, Gagne JP, Barbi de Moura M, Feinstein T, et al. ARTD1/PARP1 negatively regulates glycolysis by inhibiting hexokinase 1 independent of NAD+ depletion. Cell reports. 2014;8(6):1819-1831.

15. Xu K, Song X, Chen Z, Qin C, He Y, Zhan W. XRCC2 promotes colorectal cancer cell growth, regulates cell cycle progression, and apoptosis. Medicine. 2014;93(28):e294.

16. Ren H, Chen Z, Yang L, Xiong W, Yang H, Xu K, et al. Apolipoprotein C1 (APOC1) promotes tumor progression via MAPK signaling pathways in colorectal cancer. Cancer management and research. 2019;11:4917-4930.

17. Li J, Zhang N, Song LB, Liao WT, Jiang LL, Gong LY, et al. Astrocyte elevated gene-1 is a novel prognostic marker for breast cancer progression and overall patient survival. Clinical cancer research : an official journal of the American Association for Cancer Research. 2008;14(11):3319-3326.

18. Xu K, Song X, Chen Z, Qin C, He Y. XRCC2 rs3218536 polymorphism decreases the sensitivity of colorectal cancer cells to poly(ADP-ribose) polymerase 1 inhibitor. Oncology letters. 2014;8(3):12221228.

19. Ghosh R, Roy S, Franco S. PARP1 depletion induces RIG-I-dependent signaling in human cancer cells. PloS one. 2018;13(3):e0194611. 
20. Jain PG, Patel BD. Medicinal chemistry approaches of poly ADP-Ribose polymerase 1 (PARP1) inhibitors as anticancer agents - A recent update. European journal of medicinal chemistry. 2019;165:198-215.

21. Li Y, Li S, Wu Z, Hu F, Zhu L, Zhao X, et al. Polymorphisms in genes of APE1, PARP1, and XRCC1: risk and prognosis of colorectal cancer in a northeast Chinese population. Medical oncology. 2013;30(2):505.

22. Rank L, Veith S, Gwosch EC, Demgenski J, Ganz M, Jongmans MC, et al. Analyzing structure-function relationships of artificial and cancer-associated PARP1 variants by reconstituting TALEN-generated HeLa PARP1 knock-out cells. Nucleic acids research. 2016;44(21):10386-10405.

23. Sakthianandeswaren A, Parsons MJ, Mouradov D, MacKinnon RN, Catimel B, Liu S, et al. MACROD2 Haploinsufficiency Impairs Catalytic Activity of PARP1 and Promotes Chromosome Instability and Growth of Intestinal Tumors. Cancer discovery. 2018;8(8):988-1005.

24. Sakthianandeswaren A, Parsons MJ, Mouradov D, Sieber OM. MACROD2 deletions cause impaired PARP1 activity and chromosome instability in colorectal cancer. Oncotarget. 2018;9(69):3305633058.

25. Santos JC, Funck A, Silva-Fernandes IJ, Rabenhorst SH, Martinez CA, Ribeiro ML. Effect of APE1 T2197G (Asp148Glu) polymorphism on APE1, XRCC1, PARP1 and OGG1 expression in patients with colorectal cancer. International journal of molecular sciences. 2014;15(10):17333-17343.

26. Schaaf L, Schwab M, Ulmer C, Heine S, Murdter TE, Schmid JO, et al. Hyperthermia Synergizes with Chemotherapy by Inhibiting PARP1-Dependent DNA Replication Arrest. Cancer research. 2016;76(10):2868-2875.

27. Solier S, Zhang YW, Ballestrero A, Pommier Y, Zoppoli G. DNA damage response pathways and cell cycle checkpoints in colorectal cancer: current concepts and future perspectives for targeted treatment. Current cancer drug targets. 2012;12(4):356-371.

28. Calabrese CR, Batey MA, Thomas HD, Durkacz BW, Wang LZ, Kyle S, et al. Identification of potent nontoxic poly(ADP-Ribose) polymerase-1 inhibitors: chemopotentiation and pharmacological studies. Clinical cancer research : an official journal of the American Association for Cancer Research. 2003;9(7):2711-2718.

\section{Figures}




\section{Figure 1}
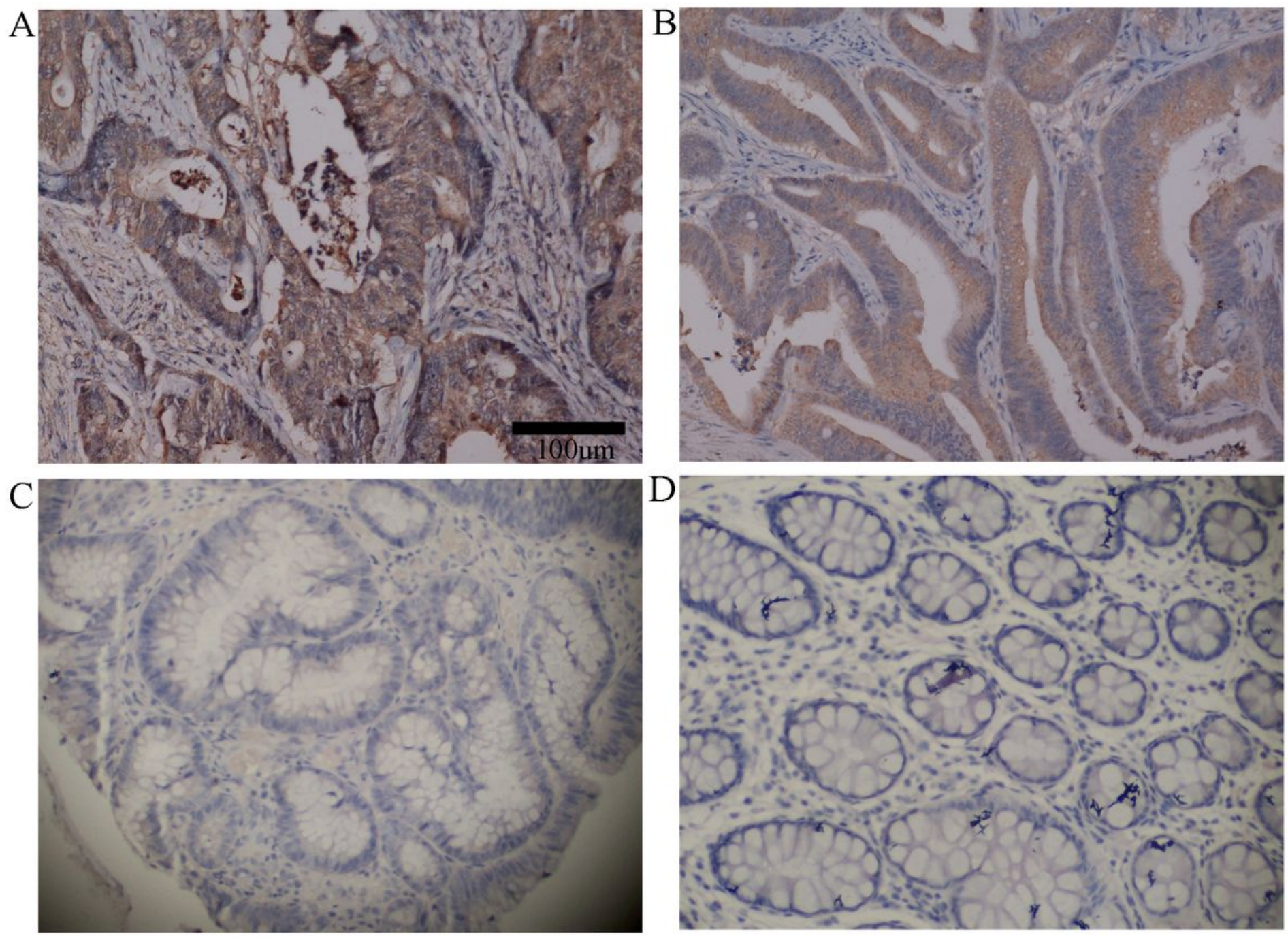

\section{Figure 1}

Expression of PARP1 in CRC and adjacent normal mucosal tissues. Primary CRC (A-C): (A) positive; (B) weakly positive (X200 magnification); (C) negative. (D) normal colorectal mucosa tissue. The scale bar represents 100um. 
Figure 2

A

Patients

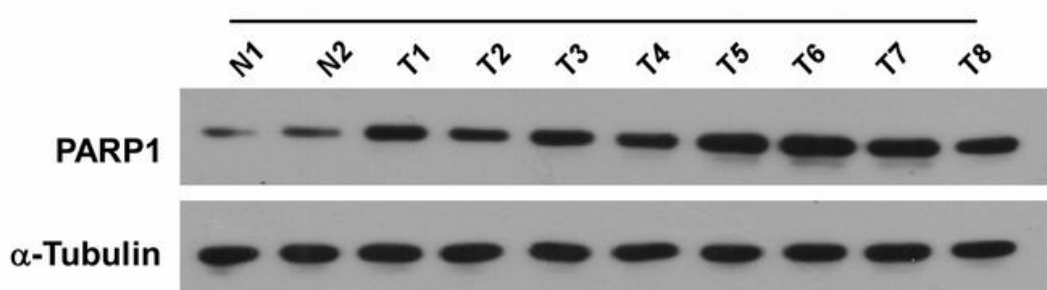

C

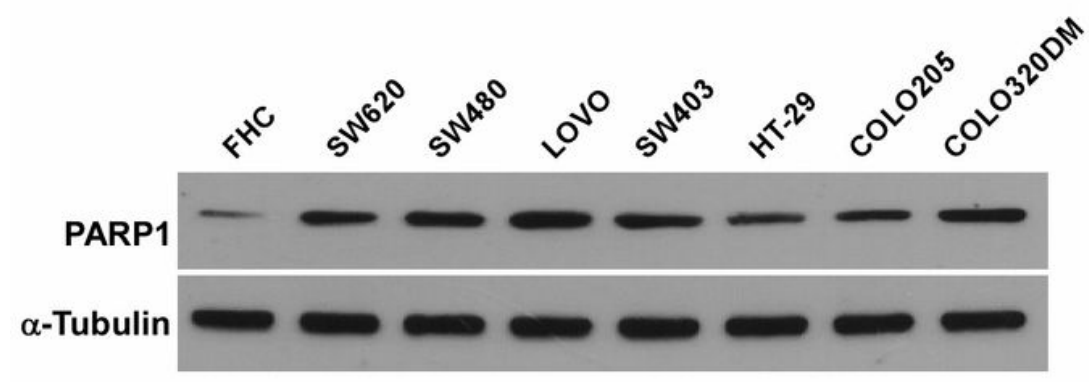

B

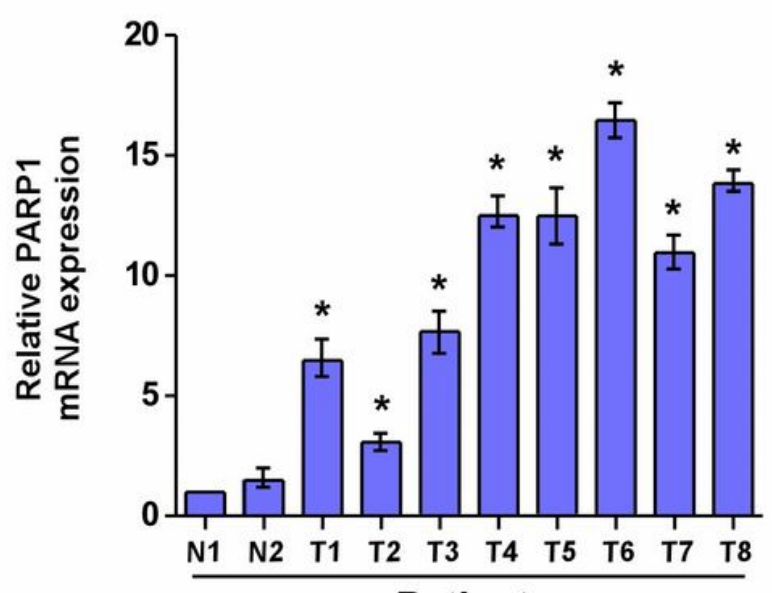

D

Patients

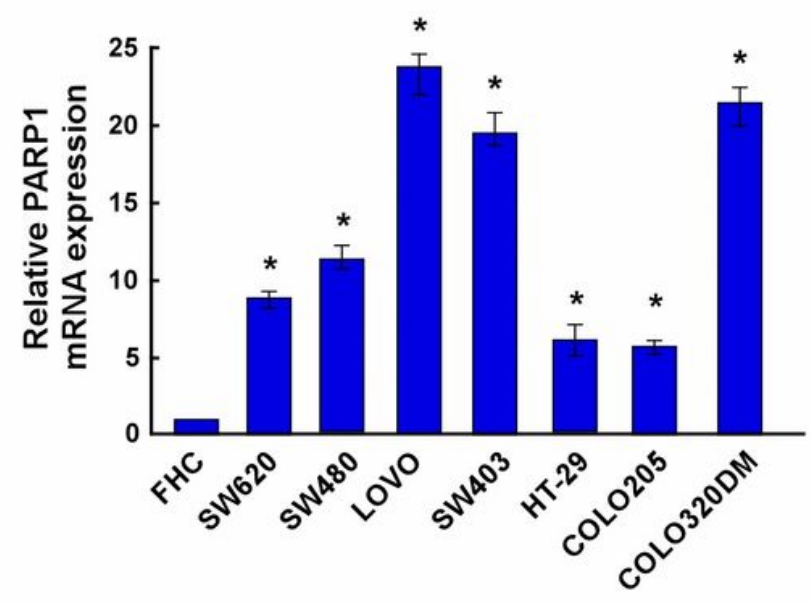

Figure 2

PARP1 expression in eight CRC tumors and matched adjacent noncancerous tissues (A-B): (A) Western blotting; (B) real-time PCR. PARP1 expression in eight CRC cell lines and normal colonic epithelial cell $(\mathrm{FHC})(\mathrm{C}-\mathrm{D})$ : (C) Western blotting; (D) real-time PCR ( $<<0.05$; Figure $2 \mathrm{C}, \mathrm{D}) .{ }^{*} \mathrm{p}<0.05$, (Student's t test). 
Figure 3
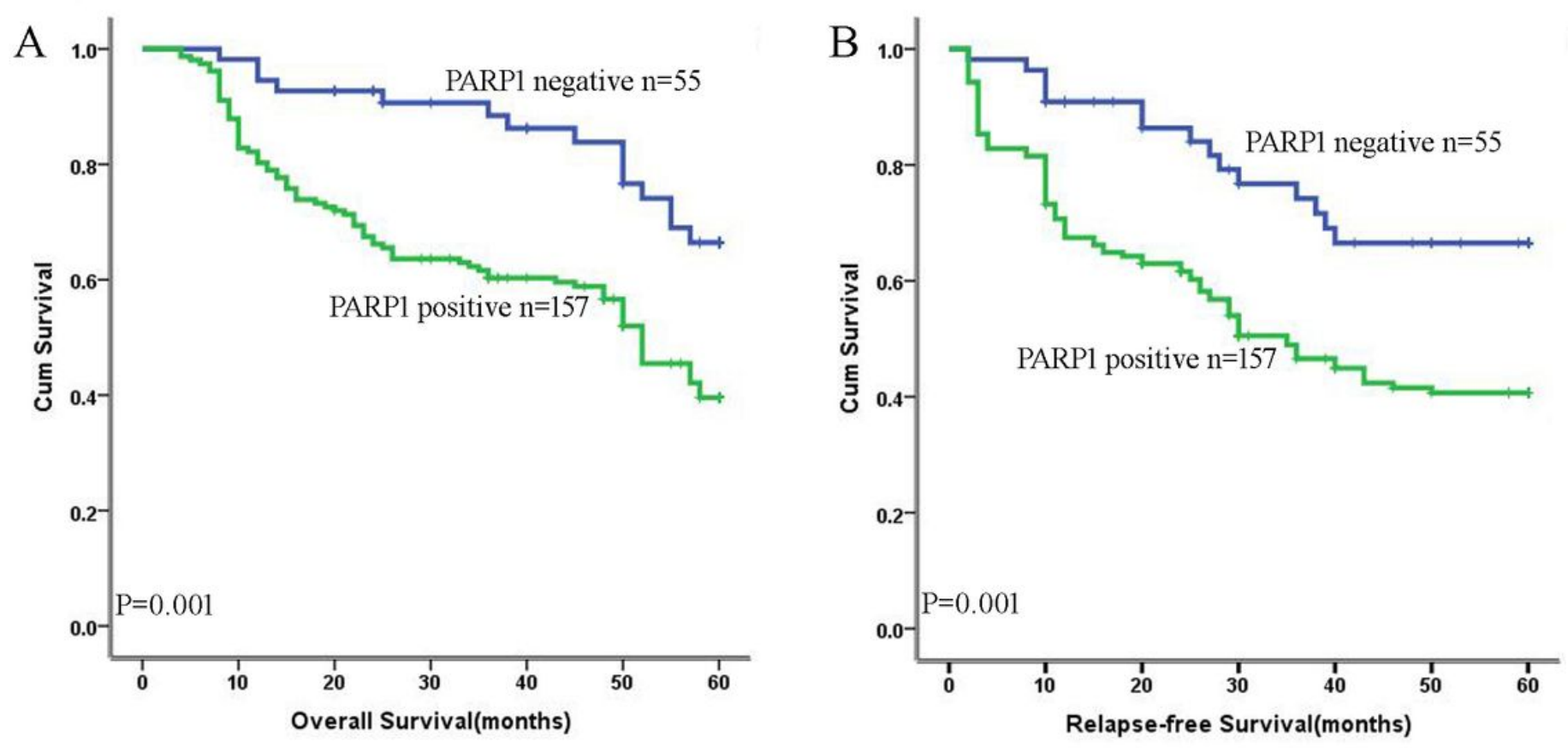

Figure 3

Survival curve in regards to PARP1 expression and the PARP1 expression in SW480/SW620 cells. Positive PARP1 expression patients have shorter OS (A) and RFS (B) than negative PARP1 expression patients ( $p$ $=0.001 ; p=0.001$, respectively).

Figure 4

A

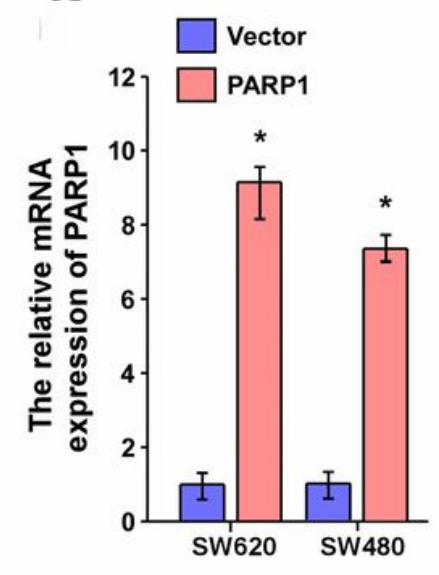

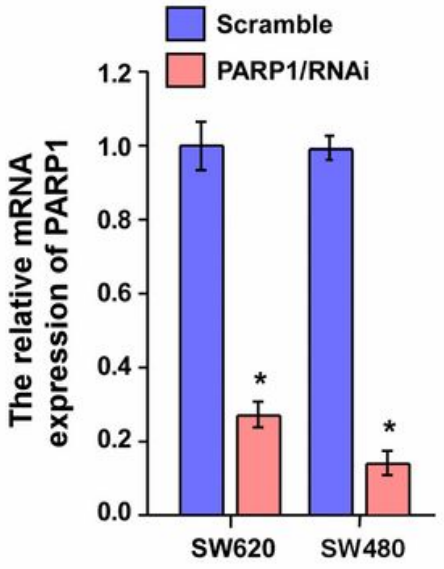

B

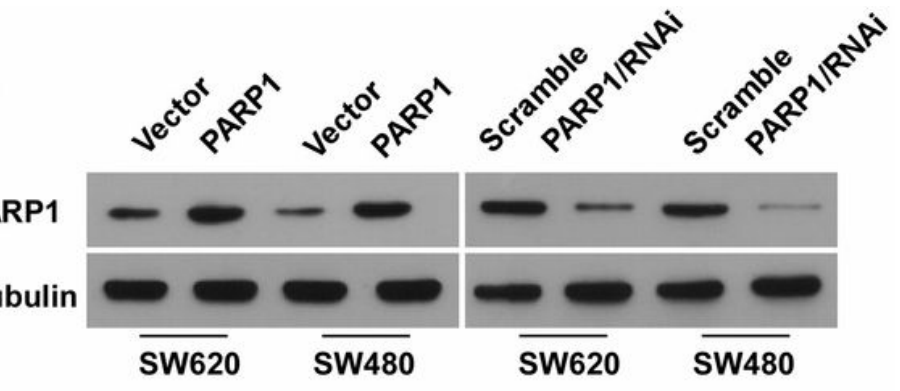

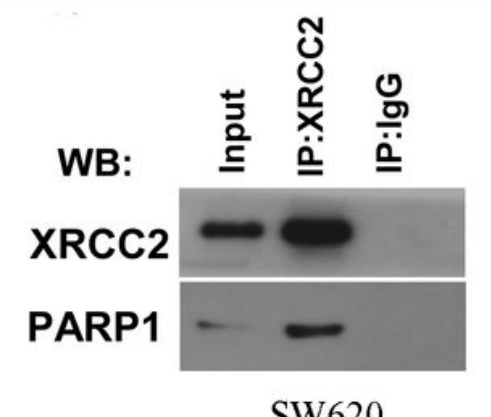

SW620

Figure 4

(A) QPCR analysis of PARP1 mRNA expression in SW480 and SW620 cells. ${ }^{*} p<0.05$, (Student's t test).

(B)Western blotting analysis of PARP1 expression in SW480/SW620-PARP1,SW480/SW620-Vector, 
SW480/SW620-PARP1/RNAi and SW480/SW620-Scramble cells. (C)The IP results of XRCC2 protein interacting with PARP1 protein. Input represents the positive control group, IgG represents the negative control group, IP represents the target experimental group.

Figure 5
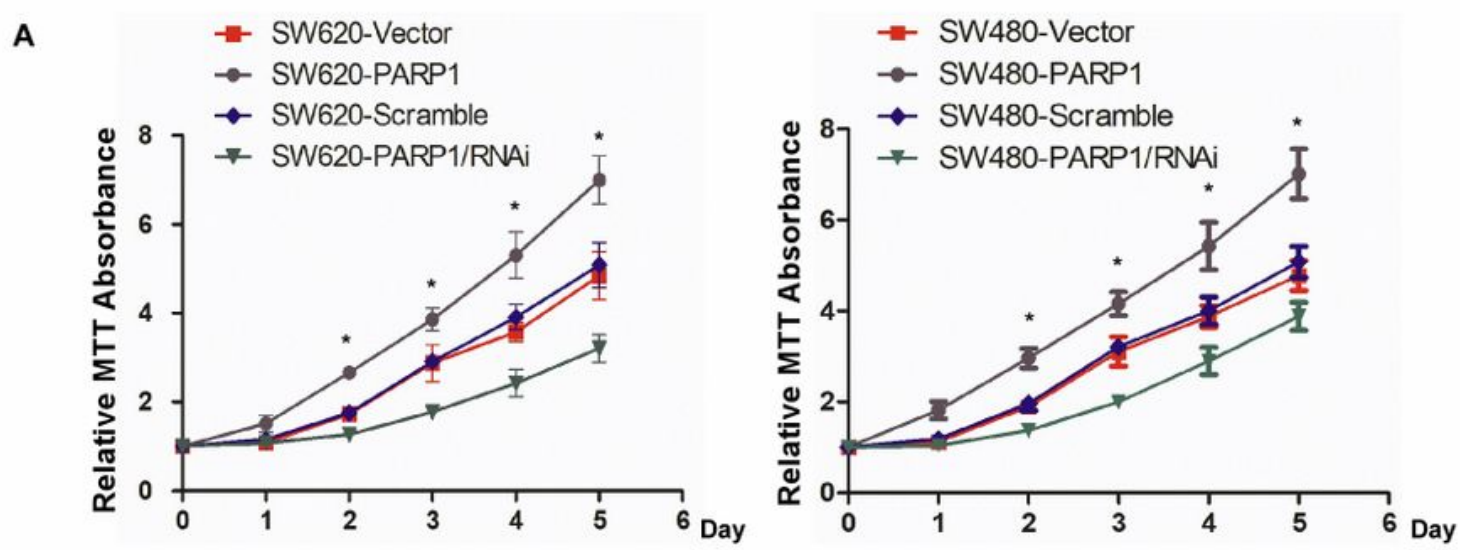

B

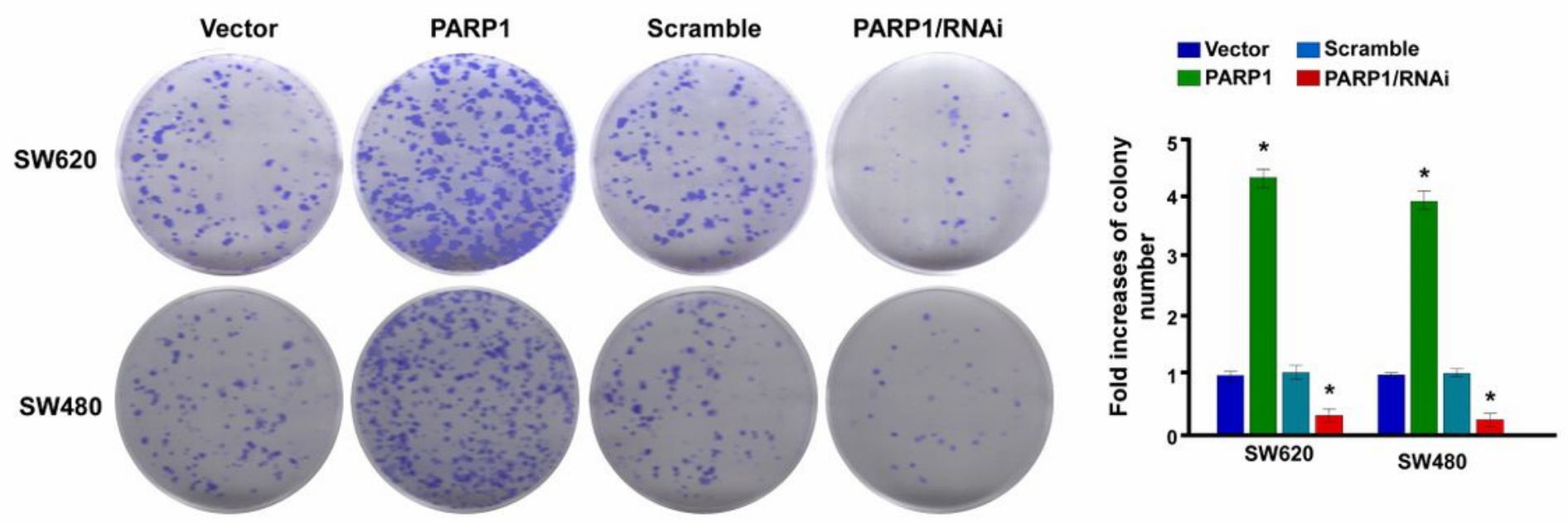

C

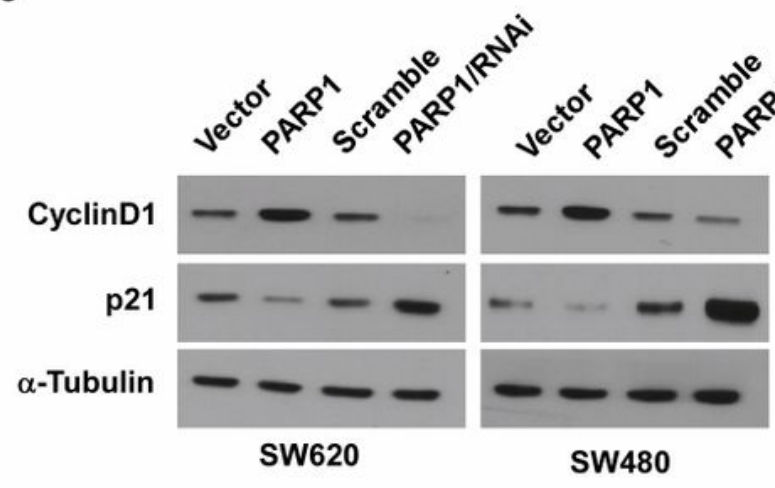

D

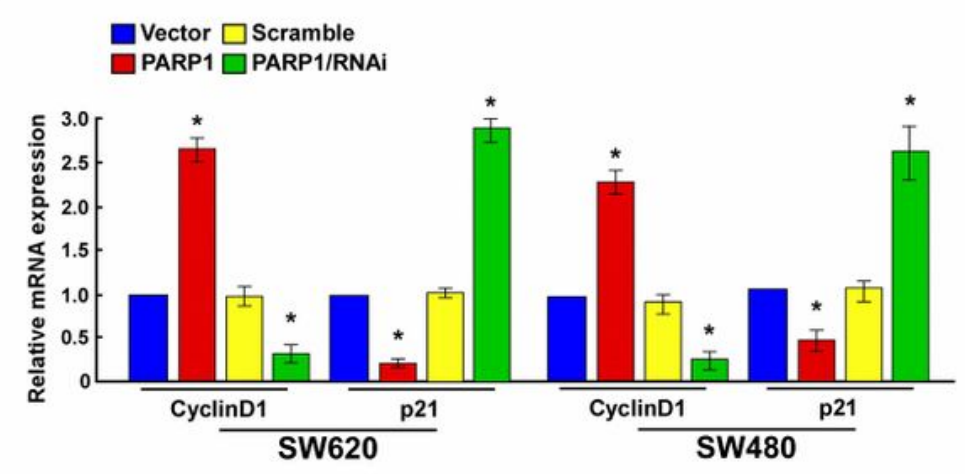

Figure 5

Cell proliferation analysis. CCK-8 assay (A) and Colony formation assay (B) of SW480/SW620 cells transfected with PARP1, PARP1/RNAi, and Vector/Scramble. Cyclin D1 and p21 expression (C-D): (C) 
Western blot analysis; (D) QPCR analysis. ${ }^{*} \mathrm{p}<0.05$, (Student's t test). 